\title{
A Review on Energy Efficient Green Communication
}

\author{
Anjuri Kulkarni ${ }^{1}$, Aarushi Gautam², Mayank Kothari ${ }^{3}$, Sachin Saonawane ${ }^{4 *}$ \\ ${ }^{1,2}$ B.Tech student at NMIMS, Mukesh Patel School of Technology Management and Engineering, Shirpur \\ ${ }^{3,4}$ Faculty at NMIMS, Mukesh Patel School of Technology Management and Engineering, Shirpur
}

*Corresponding Author: Sachin Saonawane, Faculty at NMIMS, Mukesh Patel School of Technology

Management and Engineering, Shirpur, India.

\begin{abstract}
Wireless communication has reached new heights with the increasing subscriber demands thus leading to emerging of environmental problems. It is becoming challenging to maintain the required quality of service in radio frequency communication. The temporary solution adapted by service providers to address the critical issues in radio communication including co-channel interference, adjacent channel interference, crosstalk etc. is to increase the power level of transmitted signal. But at the same time, such high power signals may affect living species badly and hence it is required to look forward for an alternate solution to high power signal communication. Green communication is capable to offer an effective solution for addressing these issues. It's mitigation to the problem of $\mathrm{CO} 2$ emission and better performance has drawn researcher's attention in past few years. It is also being thought as an energy efficient communication. The upcoming $5 G$ networks are undergoing standardization and need to have least power consumption. This paper deals with reviews about the challenges and opportunities in establishing green communication and various key techniques.
\end{abstract}

Keywords: energy efficiency, wireless spectral efficiency, 5G, MIMO, IOT

\section{INTRODUCTION}

With the rapid growth of wireless communication networks, the energy consumption is also increasing at a fast rate. In order to provide improved data rate and better quality of service, service providers have led to a matter of concern over the energy efficiency. Researchers conclude that mobile operators are among the top energy consumers. The evolution from first and second generation networks to third and fourth generation networks was to improve capacity and $5 \mathrm{G}$ is currently in embryonic stage and will unravel by 2020. The energy efficiency of $5 \mathrm{G}$ networks is expected to increase 100x times from $1000 \mathrm{~mW} / \mathrm{Mbps} / \mathrm{sec}$ to $10 \mathrm{~mW} / \mathrm{Mbps} / \mathrm{sec}$ in future [7].

With the increasing energy cost and power consumption, wireless communication has led to various environmental problems. Thus green communication is an urgent need. Green Communication ambitions at improving the strength efficiency and lowering the electricity intake and emission of $\mathrm{CO} 2$ [5]. Wireless networking technologies with enhanced concept of cognitive radio for spectrum utilization will play a major role in green communication. However, it is contradicting the fact that the wireless verbal exchange infrastructures, just like the infrastructures controlled by way of cellular operators play a major role inside the ever-growing strength costs [1] [14].

This article first analyzes the need of 'green solutions', then the various challenges it is facing and thus provides the key techniques that will consequently help in reducing the environmental problems due to human activities.

\section{NEED FOR GREEN COMMUNICATION}

With the over-rising demands of electronic calling gadgets and software driven smart phones, the internet is now available to all mobile users thus increasing the demand for broadband [8]. For fixing the difficulty, 1,20,000 Base stations are deployed each year for servicing all of the new mobile smartphone subscriber across the world.

In OSI version the wonderful separation among the layers creates a barrier to transformational upgrades that's unsettled presently. A layout which integrates the complete verbal exchange community from physical layer through worldwide conversation system needs to be carried out [10]. 


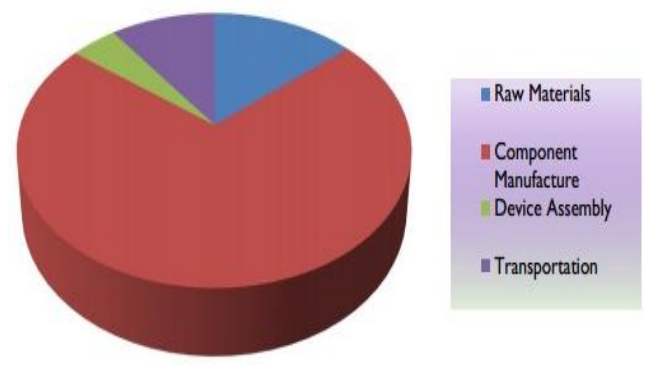

Figure1. Mobile device Supply chain emissions split by production Stage 2019 [courtesy: Jupiter research]

\section{Challenges in Green Communication}

With the evolution of present communique gadget, we also are handling the cutting-edge issues like development and optimization of communique centers. With huge benefits, green communication comes at a price. Some of the main challenges of green solutions are as follows:

\section{- Cost}

Although green communication has less energy consumption but still it has much higher cost than the existing techniques. The incorporation of green solutions in devices leads to increase in their price [6].

\section{- Spectrum Efficiency}

It is defined as throughput of the system. According to Shannon's capacity formula the transmission rate is directly proportional to the amount of available transmit power and bandwidth. [2]. the data rate would be impacted in order to control the transmission power to make the communication green.

\section{- Bandwidth}

As previously stated that the transmission rate is directly proportional to bandwidth, for a given transmission rate, the energy consumption can be reduced by expanding the bandwidth [3]. However, green communication will not necessarily be favorable in this case, as bandwidth expansion requires changes in the existing networks.

"Green communication requires large energy consumption equipments to remain updated with the changing technologies" [1]. To save energy and reduce the emission of $\mathrm{CO} 2$ following aspects can be considered: On one hand, strength-saving may be completed through optimizing product design, which includes lessen the thickness of unmarried and over-thick PCB board, enhancing the air flow and heat dispersion. Then again, considering chips and electronic gadgets eat most of the electricity of the product it's miles critical to decrease the electricity intake [5].

\section{Key TEChNiQUeS OF GREen COMMUNiCATION}

The existing solutions for green communication are the following:

\section{- Cognitive Network}

Inexperienced spectrum useful resource management is a critical part of green communication. The spectrum aid usage performance and the community transmission overall performance may be efficaciously progressed by way of the cognitive network era [4]. Cognitive radio turned into first off provided by using Joseph Mitola who defined it as 'a system that's inexperienced of improving the nonpublic wi-fi service flexibility through radio expertise illustration language (RKRL)'. Cognitive radio includes spectrum sensing, community cognizance, vicinity attention, customers want, state, language and safety policy amongst which spectrum sensing has been most regarded by people [11] [15].

\section{- Network Coding}

Initially, the network coding was provided by in Chinese language [7]. By removing pointers the network throughput is enhanced and it could also save power consumption and bandwidth. Intermediate nodes may be made to take part in statistics dealing with by using community coding to keep away from statistics clash. Community coding uses distributed asynchronous set of regulations in multisource cycle 
broadcasting community so that it will follow network coding era in it to cast off the headaches in coding and interpreting if you need to keep the community bandwidth and ameliorate using hyperlink. Microsoft cooperation has used community coding as its core era. With the ongoing trends in community coding it is genuinely going to be a trending era in the close to destiny.

\section{- Smart Grid}

Smart grid is an aggregate of modern-day new fabric era, hardware era, automatic controlling era, laptop software, sensor technology, excessive temperature awesome engaging in era, statistics generation, strength and digital device era, allotted technology and contemporary communiqué generation. These types of technologies work collectively which will provide current strength grid with superior strength facilities of measuring and to upgrade the conventional grid with the aid of constructing a greater cozy, dependable, monetary and eco-friendly grid [6].

As steady with the definition and sort provided by using nation energy technology Laboratory the key assisting generation for clever grid may be classified into the following four categories:

1) Integrated Communication Technology

2) Sensing, Metrology and Measurement techniques

3) Advanced Grid equipment

4) Advanced Controlling system

With the intention to installation an immoderate-pace, duplex, actual-time and covered communication system smart grid needs to be determined out

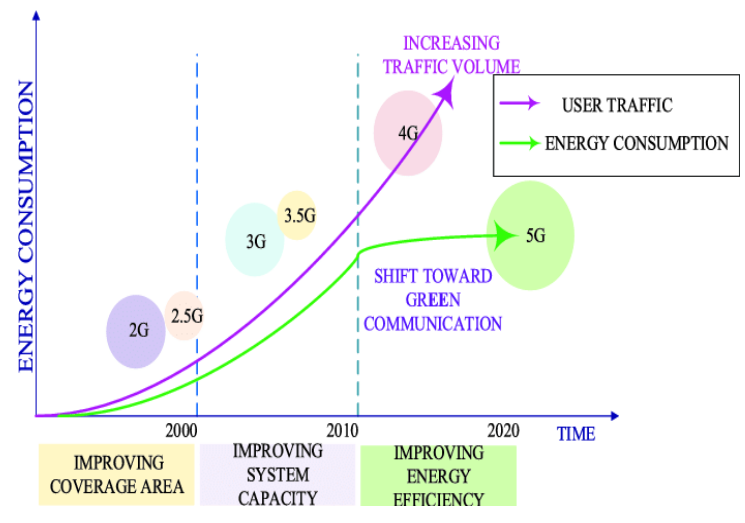

Figure1. Shift toward green communication [7]

The solutions on which research is currently ongoing and are about to emerge in the coming years are:

\section{- MIMO (3G/4G) and Massive MIMO(5G)}

MIMO technology has become popular for wireless data transmission and has been integrated into wireless standards like Wi-Fi and LTE. As the complexity of hardware and energy consumption of the signal processing at both transmitter and receiver ends increases, the cost of wireless communication also increases [7].

\section{- Co-operative communication (D2D communication)}

This communication system is integrated into LTE advanced standards and Wi-Max. A three node wireless network, referred to as "Three-terminal communication channel" is used in this communication system.

\section{- Space-Time wireless communication (O-STBC, STTC)}

The wireless communication standard for this system is CDMA2000 and UMTS mobile standards. Space Time Coding (STC) [9] is a wireless system that engrosses several transmitting antennas and single or many receiving antennas. Multiple antennas have the potential to increase the attainable bit rates, and this was demonstrated by the help of information theory.

UMTS depends on data collection which can be accomplished by drive testing and then extended to other cellular data services [12]. 


\section{CONCLUSION AND FUTURE ASPECTS}

With the development in information and communication technology, the demand for green communication is increasing rapidly. Other than reducing the energy requirements Green Communication also helps to reduce the emission of $\mathrm{CO} 2$ which is a threat to environment and human beings. As studies suggest that green communication has many issues and challenges in terms of cost, spectrum efficiency and bandwidth saving still further research is required to discuss these open issues effectively. The existing techniques to achieve green communication are cognitive network, network coding, Smart Grid and further research is being conducted to find out better and effective techniques.

Inside the destiny green communication gadget, cloud computing and the internet of factors will play a vital role and generally tend to become popular. Another important aspect which needs to be considered for future green communication is network security and secures power optimization.

\section{REFERENCES}

[1] Chuan, Fu, and Liu Anqing. "Key techniques in green communication." 2011 International Conference on Consumer Electronics, Communications and Networks (CECNet). IEEE, 2011.

[2] Ahmed Malik, Nabeel, and Masood Ur Rehman. "Green communications: Techniques and challenges." EAI Endorsed Transactions on Energy Web 2017.14 (2017).

[3] Le, Bin, Thomas W. Rondeau, and Charles W. Bostian. "Cognitive radio realities." Wireless Communications and Mobile Computing 7.9 (2007): 1037-1048.

[4] Yan, Xijin, Jun Yang, and Zhen Zhang. "An improved outer bound for multisource multisink network coding." Proc. 1st Workshop on Network Coding, Theory, and Applications (NETCOD). 2005.

[5] Zhang, Honggang. "Cognitive radio for green communications and green spectrum." Int. Conf. Commun. and Networking in China. 2008.

[6] Kumar, Ashish, Kriti Singh, and Debmalya Bhattacharya. "Green communication and wireless networking." 2013 International Conference on Green Computing, Communication and Conservation of Energy (ICGCE). IEEE, 2013.

[7] Abrol, Akshita, and Rakesh Kumar Jha. "Power optimization in 5G networks: A step towards GrEEn communication." IEEE Access 4 (2016): 1355-1374.

[8] Kalyani, T., Anuradha Thotakuri, and Mahendra Vucha. "Review on Energy Efficient Green Communication Networks for Wireless and Mobile Communication." (2006).

[9] Ahlswede, Rudolf, et al. "Network information flow." IEEE Transactions on information theory 46.4 (2000): 1204-1216.

[10] Björnson, Emil, Marios Kountouris, and Mérouane Debbah. "Massive MIMO and small cells: Improving energy efficiency by optimal soft-cell coordination." ICT 2013. IEEE, 2013.

[11] Chiaraviglio, Luca, et al. "Energy-efficient management of UMTS access networks." 2009 21st International Teletraffic Congress. IEEE, 2009.

[12] Jayaweera, Sudharman K. "Virtual MIMO-based cooperative communication for energy-constrained wireless sensor networks." IEEE Transactions on Wireless communications 5.5 (2006): 984-989.

[13] Han, Congzheng, et al. "Green radio: radio techniques to enable energy-efficient wireless networks." IEEE communications magazine 49.6 (2011): 46-54.

[14] Arjoune, Youness, and Naima Kaabouch. "A comprehensive survey on spectrum sensing in cognitive radio networks: Recent advances, new challenges, and future research directions." Sensors 19.1 (2019): 126.

[15] Kusaladharma, Sachitha, and Chintha Tellambura. "An overview of cognitive radio networks." Wiley Encyclopedia of Electrical and Electronics Engineering (1999): 1-17.

Citation: Sachin Saonawane, et.al, "A Review on Energy Efficient Green Communication”, International Journal of Innovative Research in Electronics and Communications, 7(2), pp. 8-11. DOI: http://dx.doi.org/10.20431/2349-4050.0702002

Copyright: () 2020 Authors, This is an open-access article distributed under the terms of the Creative Commons Attribution License, which permits unrestricted use, distribution, and reproduction in any medium, provided the original author and source are credited. 